\title{
Uniform Exponential Stabilization for Flexural Vibrations of a Solar Panel
}

\author{
Prasanta Kumar Nandi ${ }^{1}$, Ganesh Chandra Gorain ${ }^{2}$, Samarjit Kar ${ }^{1}$ \\ ${ }^{1}$ Department of Mathematics, National Institute of Technology, Durgapur, India \\ ${ }^{2}$ Department of Mathematics, J. K. College, Purulia, India \\ E-mail: goraing@gmail.com, pknandi.math@gmail.com \\ Received February 16, 2011; revised March 1, 2011; accepted March 4, 2011
}

\begin{abstract}
Here we study a problem of stabilization of the flexural vibrations or transverse vibrations of a rectangular solar panel. The dynamics of vibrations is governed by the fourth order Euler-Bernoulli beam equation. One end of the panel is held by a rigid hub and other end is totally free. Due to attachment of the hub, its dynamics leads to a non-standard equation. The exponential stabilization of the whole system is achieved by applying an active boundary control force only on the rigid hub. The result of uniform stabilization is obtained by means of an explicit form of exponential energy decay estimate.
\end{abstract}

Keywords: Solar Panel, Hybrid System, Flexural Vibrations, Uniform Exponential Stabilization, Energy Decay Estimate

\section{Introduction}

Motivated by ambitious space programs, mathematical investigation on stabilization of vibrating space structures is an active area of research among others. The pioneering work was first started since early seventies in a study aimed at achieving energy decay rates for wave equation exterior to a bounded obstacle. Later the idea has been extended in various mathematical problems related to the vibrations of flexible structures like beams, plates or slender elements capable of withstanding finite deformation. The most important problem for these problems is to suppress the vibrations to assure a good performance of the overall system.

During the last few decades, the use of flexible structures is on the rise. The vibrations of flexible structures are usually non-linear in practice. The vibrations of flexible structures are the problem of a dynamical system mathematically governed by partial differential equations, particularly, the second order wave equation and the fourth-order Euler-Bernoulli beam equation. Stabilization for the wave equation in a bounded domain have been investigated by several authors (cf. G. Chen [1,2], J. Lagnese [3,4], J. L. Lions [5], V. Komornik [6] and the references therein). Similarly, those governed by the fourth-order Euler-Bernoulli beam equation have been treated by G. Chen, and J. Zhou [7], O. Morgul [8] and A.
M. Krall [9]. Hybrid system of flexible structures consists of a coupled elastic part and a rigid part. The hybrid system in which a lumped mass is present at one end, have been treated earlier by G. Chen, M. C. Delfour, A. M. Krall and G. Payre [10], W. Littman, L. Markus [11] and B. Rao [12]. The most usual practical approach to stabilize the problems of such type is to apply the control force or the stabilizer on the free end of the elastic part instead of the rigid part.

The mathematical theory of stabilization of distributed parameter system has become a great interest in view of its application in various flexible structures. The energy decay estimate has earlier been studied by several authors (cf. G. Chen [1,2], J. Lagnese [3,4], J. L. Lions [5], V. Komornik and E. Zuazua [13]). Recently, G. C. Gorain [14] treated the case of internally damped wave equations for the so called Kelvin-Voigt model of viscoelsticity together with undamped boundary conditions (without considering any boundary feedback) to obtain a uniform exponential energy decay estimate. Several hybrid model of the dynamics of torsional vibrations of a flexible structure hoisted by a rigid hub at one end have been studied by T. Fukuda, F. Arai, H. Hosogai and N. Yajima [15]. In engineering literature, a common approach to treat the above problems is to decompose the vibrations into normal modes and retain the first few modes to reduce the problem into a finite dimensional 
state space representation. The question of uniform stabilization or point-wise stabilization of Euler-Bernoulli beams or serially connected beams has been studied by a number of authors (cf. J. L. Lions [5], G. Chen, M. C. Delfour, A. M. Krall and G. Payre [10], K. Ammari and M. Tuesnak [16], K. Liu and Z. Liu [17], K. Nagaya [18], R. Rebarbery [19] etc.).

\section{Mathematical Formulation of the Problem}

We consider a uniform rectangular flexible solar panel hoisted by a rigid hub at one end. The panel is of length $L$, unit width, having uniform mass density $m$ per unit length, which is rigidly attached by a lumped mass $m_{h}$ (hub) at one end and that is totally free at the other end. Our aim is to stabilize the vibrations of the overall system by applying a suitable stabilizer or damper on the rigid hub, when it is initially set in motion.

Referring to the schematic Figure 1, if $y_{h}(t)$ is the transverse displacement of the rigid hub and $y_{p}(x, t)$ is that of the panel at the position $x$ along the span of the panel relative to the hub at time $t$, then the total transverse deflection can be written as

$$
y(x, t)=y_{h}(t)+y_{p}(x, t), 0 \leq x \leq L, t \geq 0 .
$$

Let us assume that the vibrations undergo only small deformations, that means, $|y(x, t)| \ll L$ and $\left|\frac{\partial y}{\partial x}(x, t)\right| \ll 1$, and neglect the gravitational effect and rotatory inertia of the panel cross sections. Then $y(x, t)$ satisfies fourth order Euler-Bernoulli beam equation

$$
m \frac{\partial^{2} y}{\partial t^{2}}(x, t)+D \frac{\partial^{4} y}{\partial x^{4}}(x, t)=0,0 \leq x \leq L, t \geq 0
$$

where $D=\frac{1}{12} E h^{3}\left(1-v^{2}\right)^{-1}$. The constants $D, E, v$ and $h$ are the flexural rigidity, the Youngs's modulus, the Poisson's ratio and the thickness of the panel respectively.

The dynamics at the hub end $x=0$, where a control force $Q(t)$ is applied, yields the differential equation

$$
m_{h} \frac{\partial^{2} y_{h}}{\partial t^{2}}(t)+D \frac{\partial^{3} y_{p}}{\partial x^{3}}(0, t)+Q(t)=0, t \geq 0 .
$$

The Equation (3) is not a standard Dirichlet or Neumann boundary condition, that is generally found in the several works of this subject area. Again since $y_{p}(0, t)=0$, it follows from (1) that $y(0, t)=y_{h}(t)$ and also we have $\frac{\partial y}{\partial x}(x, t)=\frac{\partial y_{p}}{\partial x}(x, t)$. Hence the Equation (3) becomes

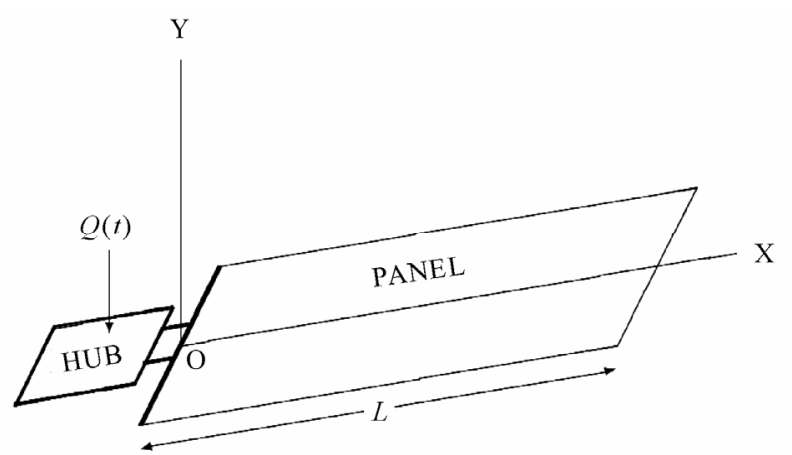

Figure 1. Schematic of the solar panel with rigid hub.

$$
\frac{\partial^{3} y}{\partial x^{3}}(0, t)+\alpha \frac{\partial^{2} y}{\partial t^{2}}(0, t)+\lambda Q(t)=0, t \geq 0,
$$

where $\alpha=\frac{m_{h}}{D}$ and $\lambda=\frac{1}{D}$. Assuming at $x=0$, there is no rotational deflection of the panel relative to the hub (that means, the panel is built in position with hub at $x=0)$, we have $\frac{\partial y_{p}}{\partial x}(0, t)=0$, implying

$$
\frac{\partial y}{\partial x}(0, t)=0, t \geq 0
$$

Since the panel is assumed to be free at $x=L$, so at this end

$$
\frac{\partial^{2} y}{\partial x^{2}}(L, t)=0 \text { and } \frac{\partial^{3} y}{\partial x^{3}}(L, t)=0, t \geq 0
$$

Let the panel be set to vibrations with arbitrary initial values

$$
y(x, 0)=y_{0}(x) \text { and } \frac{\partial y}{\partial t}(x, 0)=y_{1}(x), 0 \leq x \leq L .
$$

Therefore, the mathematical model to be studied for flexural or transverse vibrations of a uniform rectangular flexible solar panel with a rigid hub at one end, is governed by the following system of equations :

$$
\begin{gathered}
m \frac{\partial^{2} y}{\partial t^{2}}(x, t)+D \frac{\partial^{4} y}{\partial x^{4}}(x, t)=0,0 \leq x \leq L, t \geq 0 \\
\frac{\partial^{3} y}{\partial x^{3}}(0, t)+\alpha \frac{\partial^{2} y}{\partial t^{2}}(0, t)+\lambda Q(t)=0 \text { and } \frac{\partial y}{\partial x}(0, t)=0 \\
\frac{\partial^{2} y}{\partial x^{2}}(L, t)=0 \text { and } \frac{\partial^{3} y}{\partial x^{3}}(L, t)=0, t \geq 0 \\
y(x, 0)=y_{0}(x) \text { and } \frac{\partial y}{\partial t}(x, 0)=y_{1}(x), 0 \leq x \leq L
\end{gathered}
$$

Because of the damping character of the control force 
$Q(t)$, it must be an odd function of velocity, that means,

$$
Q(t)=f\left(\frac{\partial y}{\partial t}(0, t)\right)
$$

where $f$ is an odd function of its argument such that $f(0)=0$ and $u f(u)>0$ for every $u \in \mathbb{R}-\{0\}$. For example, if $f(u)=u$, we have a simple viscous damper.

\section{Energy of the System}

The total energy $E(t)$ at time $t$ is defined by

$$
E(t)=\frac{1}{2} \int_{0}^{L}\left[m\left(\frac{\partial y}{\partial t}\right)^{2}+D\left(\frac{\partial^{2} y}{\partial x^{2}}\right)^{2}\right] \mathrm{d} x+\frac{1}{2} m_{h}\left[\frac{\partial y}{\partial t}(0, t)\right]^{2}
$$

for $t \geq 0$.

Differentiating this with respect to $t$ and using the governing Equations (8)-(10), we obtain

$$
\begin{aligned}
\frac{\mathrm{d} E}{\mathrm{~d} t}= & c^{2} \int_{0}^{L}\left[m \frac{\partial y}{\partial t} \frac{\partial^{2} y}{\partial t^{2}}+D \frac{\partial^{2} y}{\partial x^{2}} \frac{\partial^{3} y}{\partial x^{2} \partial t}\right] \mathrm{d} x \\
& +m_{h} \frac{\partial y}{\partial t}(0, t) \frac{\partial^{2} y}{\partial t^{2}}(0, t) \\
= & D\left[\frac{\partial y}{\partial t}(0, t) \frac{\partial^{3} y}{\partial x^{3}}(0, t)+\alpha \frac{\partial y}{\partial t}(0, t) \frac{\partial^{2} y}{\partial t^{2}}(0, t)\right] \\
= & -D \lambda \frac{\partial y}{\partial t}(0, t) Q(t),
\end{aligned}
$$

where the integration is performed by parts and the boundary conditions (9)-(10) are used. By the help of the Equation (12), we get

$$
\frac{\mathrm{d} E}{\mathrm{~d} t}=-\frac{\partial y}{\partial t}(0, t) f\left(\frac{\partial y}{\partial t}(0, t)\right)=-u f(u)<0 \text { for } t \geq 0,(15)
$$

where $u=\frac{\partial y}{\partial t}(0, t)$ is the velocity at the hub end and $D \lambda=1$. The result (15) implies that the energy $E(t)$ of the system (8)-(12) is a non increasing function of time. Integrating (15), we have $E(t) \leq E(0)$, where

$$
E(0)=\frac{1}{2} \int_{0}^{L}\left[m\left(y_{1}(x)\right)^{2}+D\left(y_{0}^{\prime \prime}(x)\right)^{2}\right] \mathrm{d} x+\frac{1}{2} m_{h}\left(y_{1}(0)\right)^{2}
$$

is the initial energy of the system. As the energy decays, our main interest is whether this decays is uniformly exponential or not. An affirmative answer can be found in the next section.

\section{Uniform Stability Result and Proof}

The main result of this paper can be stated in the following theorem.

Theorem 1. Let $y(x, t)$ be a solution of the system (8)-(12) with the initial values $\left\{y_{0}, y_{1}\right\}$ for which $E(0)<+\infty$, where $E(0)$ is defined in (16). Then the total energy of the system decays uniformly exponentially with time, that means, $\forall t \geq 0, E(t)$ satisfies the relation

$$
E(t) \leq M \mathrm{e}^{-\mu t}
$$

for some finite reals $M>1$ and $\mu>0$, both being independent of the time $t$.

The theorem will be proved after some preliminary steps. First, we require the following inequality.

For any real number $\alpha>0$, we have by the CauchySchwartz's inequality

$$
|f \cdot g| \leq \frac{1}{2 \alpha}\left(|f|^{2}+\alpha^{2}|g|^{2}\right) .
$$

Next we consider the following lemma:

Lemma 1. For every solution $y(x, t)$ of the system (8)-(12), the time derivative of the functional $G$ (cf. G. C. Gorain [20], G. C. Gorain and S. K. Bose [21]) defined by

$$
G(t)=m \int_{0}^{L} x \frac{\partial y}{\partial t} \frac{\partial y}{\partial x} \mathrm{~d} x \text { for } t \geq 0
$$

satisfies

$$
\frac{\mathrm{d} G}{\mathrm{~d} t} \leq 12 L m\left[\frac{\partial y}{\partial t}(L, t)\right]^{2}+\frac{1}{2} m_{h}\left[\frac{\partial y}{\partial t}(0, t)\right]^{2}-E(t) .
$$

Proof: If we differentiate (19) with respect to $t$ and using the governing Equation (8), we obtain

$$
\begin{aligned}
\frac{\mathrm{d} G}{\mathrm{~d} t}= & \int_{0}^{L} x\left(m \frac{\partial y}{\partial t} \frac{\partial^{2} y}{\partial x \partial t}-D \frac{\partial y}{\partial x} \frac{\partial^{4} y}{\partial x^{4}}\right) \mathrm{d} x \\
= & \frac{1}{2} L m\left[\frac{\partial y}{\partial t}(L, t)\right]^{2}+\frac{1}{2} m_{h}\left[\frac{\partial y}{\partial t}(0, t)\right]^{2} \\
& -E(t)-D \int_{0}^{L}\left(\frac{\partial^{2} y}{\partial x^{2}}\right)^{2} \mathrm{~d} x,
\end{aligned}
$$

where the integration is done by parts and the boundary conditions (9)-(10) are used. The Lemma 1 then follows immediately from (21).

Proof of Theorem 1: Proceeding as in G. C. Gorain [20] and G. C. Gorain and S. K. Bose [21], we define energy like Lyapunov functional $V$ by

$$
V(t)=E(t)+\varepsilon G(t) \text { for } t \geq 0,
$$

where $\varepsilon>0$ is a fixed constant. Differentiating (22) with respect to $t$, and using (15) and (21) we obtain 


$$
\begin{aligned}
\frac{\mathrm{d} V}{\mathrm{~d} t} \leq & -\frac{\partial y}{\partial t}(0, t) f\left(\frac{\partial y}{\partial t}(0, t)\right)+\varepsilon \frac{1}{2} \operatorname{Lm}\left[\frac{\partial y}{\partial t}(L, t)\right]^{2} \\
& +\varepsilon \frac{1}{2} m_{h}\left[\frac{\partial y}{\partial t}(0, t)\right]^{2}-\varepsilon E(t) .
\end{aligned}
$$

We choose a feedback controller or stabilizer in such way that satisfies mathematically

$$
\begin{aligned}
& \frac{1}{2} \operatorname{Lm}\left(\frac{\partial y}{\partial t}(L, t)\right)^{2}+\frac{1}{2} m_{h}\left(\frac{\partial y}{\partial t}(0, t)\right)^{2} \\
& \leq \beta \frac{\partial y}{\partial t}(0, t) f\left(\frac{\partial y}{\partial t}(0, t)\right),
\end{aligned}
$$

where $\beta$ is a finite positive constant independent of time $t$.

Hence, using the above relation (24), we can write (23) as

$$
\frac{\mathrm{d} V}{\mathrm{~d} t} \leq(\beta \varepsilon-1) \frac{\partial y}{\partial t}(0, t) f\left(\frac{\partial y}{\partial t}(0, t)\right)-\varepsilon E(t) .
$$

Since $\varepsilon$ is small, we may assume that

$$
0<\varepsilon<\frac{1}{\beta}
$$

so that the differential relation (25) reduces to

$$
\frac{\mathrm{d} V}{\mathrm{~d} t}+\varepsilon E(t) \leq 0 \text { for } t \geq 0
$$

Now applying the Inequality (18), we have from (19),

$$
|G(t)| \leq \frac{2 L^{2}}{\pi} \sqrt{\frac{m}{D}} \int_{0}^{L}\left[m\left(\frac{\partial y}{\partial t}\right)^{2}+D \frac{\pi^{2}}{4 L^{2}}\left(\frac{\partial y}{\partial x}\right)^{2}\right] \mathrm{d} x,
$$

Again using Wirtinger's inequality

$$
\int_{0}^{L}\left(\frac{\partial y^{2}}{\partial x}\right) \mathrm{d} x \leq \frac{4 L^{2}}{\pi^{2}} \int_{0}^{L}\left(\frac{\partial^{2} y^{2}}{\partial x^{2}}\right) \mathrm{d} x
$$

the above Inequality (28) can be expressed as

$$
|G(t)| \leq \frac{4 L^{2}}{\pi} \sqrt{\frac{m}{D}} E(t),
$$

that means,

$$
-\frac{4 L^{2}}{\pi} \sqrt{\frac{m}{D}} E(t) \leq G(t) \leq \frac{4 L^{2}}{\pi} \sqrt{\frac{m}{D}} E(t) \text { for } t \geq 0 .
$$

So $V$ defined by (22) can be estimated as

$$
\left(1-\varepsilon \frac{4 L^{2}}{\pi} \sqrt{\frac{m}{D}}\right) E(t) \leq V(t) \leq\left(1+\varepsilon \frac{4 L^{2}}{\pi} \sqrt{\frac{m}{D}}\right) E(t) \text {. }
$$

Since $\varepsilon$ is small, we may further assume that

$$
0<\varepsilon<\frac{\pi}{4 L^{2}} \sqrt{\frac{D}{m}} .
$$

Then it follows from (32) that $V(t)>0$ for every $t \geq 0$. Invoking the Inequality (32), the relation (27) leads to the differential inequality

$$
\frac{\mathrm{d} V}{\mathrm{~d} t}+\mu V(t) \leq 0,
$$

where

$$
\mu=\frac{\varepsilon}{\left(1+\varepsilon \frac{4 L^{2}}{\pi} \sqrt{\frac{m}{D}}\right)}>0 .
$$

Multiplying (34) by $\mathrm{e}^{\mu t}$ and integrating from 0 to $t$, we obtain

$$
V(t) \leq \mathrm{e}^{-\mu t} V(0) \text { for } t \geq 0 .
$$

Applying again the Inequality (32) in (36), we get

$$
E(t) \leq M \mathrm{e}^{-\mu t} E(0),
$$

where

$$
M=\left(1+\varepsilon \frac{4 L^{2}}{\pi} \sqrt{\frac{m}{D}}\right)\left(1-\varepsilon \frac{4 L^{2}}{\pi} \sqrt{\frac{m}{D}}\right)>1 .
$$

Hence the theorem.

\section{Conclusions}

Here we have achieved the uniform boundary stabilization of flexural vibrations of a rectangular solar panel which is held by a rigid hub at one end and is totally free at the other. We have also estimated directly the exponential energy decay rate $\mu$ that is explicitly found in (35). Again, considering $\mu$ as an explicit function of $\varepsilon$, we have

$$
\frac{\mathrm{d} \mu}{\mathrm{d} \varepsilon}=\left(1+\varepsilon \frac{4 L^{2}}{\pi} \sqrt{\frac{m}{D}}\right)^{-2}>14,
$$

in view of (33). Hence the exponential decay rate as a function of $\varepsilon$ will be maximum for largest admissible value $\varepsilon$, an upper bound of which can be determined by considering the coupled relations (26) and (33) simultaneously. The determination of exact value is restricted by the lack of explicit knowledge in general of the parameter $\beta$ appearing in the literature. The motivation of considering the vibrations of this type of hybrid system arises from many practical problems such as spacecraft with flexible attachment, robot with flexible links and thin plates of different mechanical system. The significant result in this paper is that the solution of the system governed by (8)-(12) converges uniformly to zero as time $t \rightarrow+\infty$. 


\section{References}

[1] G. Chen, "Energy Decay Estimates and Exact Boundary-Value Controllability for the Wave Equation in a Bounded Domain," Journal de Mathématiques Pures et Appliquées, Vol. 58, No. 3, 1979, pp. 249-273.

[2] G. Chen, "A Note on the Boundary Stabilization of the Wave Equation," SIAM Journal on Control and Optimization, Vol. 19, No. 1, 1981, pp. 106-113. doi: $10.1137 / 0319008$

[3] J. Lagnese, "Note on Boundary Stabilization of Wave Equations," SIAM Journal on Control and Optimization, Vol. 26, No. 5, 1988, pp. 1250-1256. doi: $10.1137 / 0326068$

[4] J. Lagnese, "Decay of Solutions of Wave Equations in a Bounded Region with Boundary Dissipation," Journal of Differential Equations, Vol. 50, No. 2, 1983, pp. 163-182. doi:10.1016/0022-0396(83)90073-6

[5] J. L. Lions, "Exact Controllability, Stabilization and Perturbations for Distributed Systems," SIAM Review, Vol. 30, No. 1, 1988, pp. 1-68. doi:10.1137/1030001

[6] V. Komornik, "Rapid Boundary Stabilization of Wave Equations," SIAM Journal on Control and Optimization, Vol. 29, 1991, pp. 197-208. doi:10.1137/0329011

[7] G. Chen and J. Zhou, "The Wave Propagation Method for the Analysis of Boundary Stabilization in Vibrating Structures," SIAM Journal on Applied Mathematics, Vol. 50, No. 5, 1990, pp. 1254-1283. doi: $10.1137 / 0150076$

[8] O. Morgul, "Dynamic Boundary Control of a Euler-Bernoulli Beam," IEEE Transactions on Automatic Control, Vol. 37, No. 5, 1992, pp. 639-642. doi:10.1109/9.135504

[9] A. M. Krall, "Asymptotic Stability of the Euler-Bernoulli Beam with Boundary Control," Journal of Mathematical Analysis and Applications, Vol. 137, No. 1, 1989, pp. 288-295. doi:10.1016/0022-247X(89)90289-8

[10] G. Chen, M. C. Delfour, A. M. Krall and G. Payre, "Modelling, Stabilization and Control of Serially Connected Beams," SIAM Journal on Control and Optimization, Vol. 25, No. 3, 1987, pp. 526-546. doi: $10.1137 / 0325029$

[11] W. Littman and L. Markus, "Stabilization of a Hybrid System of Elasticity by Feedback Boundary Damping," Annali di Matematica Pura ed Applicata, Vol. 152, No. 1,
1988, pp. 281-330. doi:10.1007/BF01766154

[12] B. Rao, "Uniform Stabilization of a Hybrid System of Elasticity," SIAM Journal on Control and Optimization, Vol. 33, No. 2, 1995, pp. 440-454. doi:10.1137/S0363012992239879

[13] V. Komornik and E. Zuazua, "A Direct Method for Boundary Stabilization of the Wave Equation," Journal de Mathématiques Pures et Appliquées, Vol. 69, No. 2, 1990, pp. 33-54.

[14] G. C. Gorain, "Exponential Energy Decay Estimate for the Solutions of Internally Damped Wave Equation in a Bounded Domain," Journal of Mathematical Analysis and Applications, Vol. 216, No. 2, 1997, pp. 510-520. doi:10.1006/jmaa.1997.5678

[15] T. Fukuda, F. Arai, H. Hosogai and N. Yajima, "Torsional Vibrations Control of Flexible Space Structures," Theoretical and Applied Mechanics, Vol. 36, No. 2, 1988, pp. 285-294.

[16] K. Ammari and M. Tuesnak, "Stabilization of BernoulliEuler Beams by Means of a Point Feedback Force," SIAM Journal on Control and Optimization, Vol. 39, No. 4, 2000, pp. 1160-1181. doi:10.1137/S0363012998349315

[17] K. Liu and Z. Liu, "Exponential Decay of Energy of the Euler-Bernoulli Beam with Locally Distributed Kelvin-Voigt Damping," SIAM Journal on Control and Optimization, Vol. 36, No. 3, 1998, pp. 1096-1098. doi:10.1137/S0363012996310703

[18] K. Nagaya, "Method of Control of Flexible Beams Subject to Forced Vibrations by Use of Inertia Force Cancellations," Journal of Sound and Vibration, Vol. 184, No. 2, 1995, pp. 184-194. doi:10.1006/jsvi.1995.0311

[19] R. Rebarbery, "Exponential Stability of Coupled Beams with Dissipative Joints: A Frequency Domain Approach," SIAM Journal on Control and Optimization, Vol. 33, No. 1, 1995, pp. 1-28. doi:10.1137/S0363012992240321

[20] G. C. Gorain, "Exponential Energy Decay Estimate for the Solutions of $N$-Dimensional Kirchhoff Type Wave Equation," Applied Mathematics and Computation, Vol. 177, No. 1, 2006, 235-242. doi:10.1016/j.amc.2005.11.003

[21] G. C. Gorain and S. K. Bose, "Exact Controllability and Boundary Stabilization of Torsional Vibrations of an Internally Damped Flexible Space Structures," Journal of Optimization Theory and Applications, Vol. 99, No. 2, 1998, pp. 423-442. doi:10.1023/A:1021778428222 